\title{
Preparation and Quality Evaluation of Different Oil Blends
}

\author{
Sumit Grover $^{1}$ (D), Parveen Kumari ${ }^{1}$ (D), Anil Kumar ${ }^{2}$ (D), Anjali Soni ${ }^{1}$ (D), Shalini Sehgal ${ }^{3}$ (D), Vasudha \\ Sharma $4, *$ (iD \\ 1 Department of Food Technology, Guru Jambheshwar University of Science and Technology, Hisar, India- 125001 \\ 2 School of Bioengineering and Food Technology, Shoolini University of Biotechnology and Management Sciences, Solan, \\ India-173229 \\ 3 Department of Food Technology, Bhaskaracharya College of Applied Sciences, University of Delhi, Sector-2, Phase-I, \\ Dwarka, New Delhi -110075 \\ 4 Department of Food Technology, Jamia Hamdard, New Delhi, India - 110062 \\ * Correspondence: vasudhakatwal@gmail.com;
}

Scopus Author ID 56235919900

Received: 25.09.2020; Revised: 17.10.2020; Accepted: 18.10.2020; Published: 21.10.2020

\begin{abstract}
The aim of the study was to derive an oil composition with a balanced ratio of saturated, monounsaturated, and polyunsaturated fatty acids (SFA, MUFA, PUFA) along with improved properties and shelf life. The blends were prepared to consist of linseed oil (LO) with soybean (SBO), mustard (MO), coconut (CO), olive (OO), and sunflower oil (SFO) in various ratios, i.e., 20:80, 30:70, 40:60, 50:50, 60:40, 70:30 and 80:20 (v/v), respectively. Among all blends, fatty acid ratios (SFA:MUFA:PUFA) of 1:1.4:4.6 for LO and SBO (20:80), 1.5:1:3.1 for LO and CO (80:20), and 1:1.9:3.4 for LO and OO (80:20) were found close to the recommended intake (1:1.5:1) and showed better quality. The storage study revealed that a maximum of blends retained their quality and found fit for consumption except some of the blends (LO:SBO, LO:SFO \& LO:MO), which could be because of their higher PUFA content. The present study provides the scope of blending using commonly consuming oils to balance the overall properties of the blend. Blending is one of the economical and simple processes used to improve the nutritional, physical, and sensory quality of any oil. Based on the compositional variation in oils, a better blend could be formulated with overall improved quality.
\end{abstract}

Keywords: vegetables oils; blending; fatty acid composition; shelf life; quality.

(C) 2020 by the authors. This article is an open-access article distributed under the terms and conditions of the Creative Commons Attribution (CC BY) license (https://creativecommons.org/licenses/by/4.0/).

\section{Introduction}

Fats and oils are essential nutrients in human and animal diets, being the richest source of energy and a carrier of fat-soluble vitamins. They also provide essential fatty acids and are precursors of different hormones [1]. Varieties of vegetable oil/fat have a unique composition and play a major role in the economic, sensory, and functional properties of food and food products [2]. The compositions of various commonly used vegetable oils indicate an imbalance in their fatty acid composition and are not in tune with the recommendations of an expert committee [3]. The right combination of fatty acid in triacylglycerol (TG) of oils and fats, as suggested by nutritionists, cannot always be met from single oil. A simple and alternative method to achieve this is to formulate a physical mixture of different oils in the right proportion or to develop structured lipids (SLs) from different oils. The blending of edible oils has now been accepted by the Government of India [4]. Manufacturers are permitted to blend any two 
oils, where the proportion of one oil must be not less than 20\% [4]. Nowadays, blends of few edible oils are available in the Indian market. However, it is not yet clear whether these blended oils are a physical mixture of two oils and can provide all the perceived benefits.

Mustard, soybean, sunflower, coconut, and olive oils are some of the common cooking oils in India [5]. MO is a highly consumed oil in India, containing a high amount of erucic acid (48.5-58.2\%) [5]. Since erucic acid consumption has been associated with myocardial lipidosis in test animals; therefore, such oil is doubtful to be consumed by humans [6]. Nowadays, attempts have been made to regulate the content of erucic acid in edible oils. Hence, blending is a simple and cheap process to regulate erucic acid in vegetable oil. SBO is the second most consumed oil in India, which exhibits a good amount of linoleic acid and oleic acid along with small but significant amounts of $\alpha$-linolenic acid [7]. SFO accounts for $58 \%$ of the total edible oil produced in India. The use of SFO for frying can be considered as a healthy choice due to balanced amounts of saturated fatty acids (SFA, ca. 6\%) and monounsaturated fatty acids (MUFA, ca. 20\%), and a high content of polyunsaturated fatty acids (PUFA), which constitute 68-72\% of total fatty acids [7] which have numerous positive health potentials [8]. Among edible oils, olive oil (OO) shows important and outstanding characteristics due to its distinctive sensorial qualities (taste and flavor) and high nutritional value that has been acknowledged across the world. Several health benefits associated with its consumption were initially observed among Mediterranean people, and its dietary consumption is nowadays considered highly beneficial to human health [9-11]. OO is rich in oleic acid, which helps to decrease the level of LDL cholesterol in blood plasma, which reduces the chances of coronary heart disease. Coconut oil is a clear liquid at ambient temperature and has a pleasant aroma. It mainly consists of saturated fatty acids (>91\%), and the major portion of saturated fatty acids are mediumchain fatty acids (MCFA) (>51\%) that are easily digestible and absorbed into the body through the portal vein and generate energy [12-14] along with antibacterial and antioxidant potentials [15]. Linseed or flaxseed has been extensively studied for its numerous health benefits [16-17] but has still not occupied a space in the cooking oil market. Recently, LO has been suggested for cooking by FSSAI and ICMR as it possesses a high amount of fiber lignin, which acts as antioxidants.

The blending of LO can be done to reduce the high content of $\omega-3$ fatty acid, and as it affects the stability of the oil. As per the World Health Organization (WHO), the proportions of SFA, MUFA, and PUFA in the dietary fats should be in the proportion of 1:1.5:1 [18]. According to various investigations, the above-mentioned ratios of fatty acids are highly recommended for maintaining good health, but no natural oil possesses such a balanced composition. Hence, an attempt was made to develop an oil/fat blend that could have better chemical properties with balanced fatty acid composition.

\section{Materials and Methods}

The present investigation was designed to evaluate the fatty acid composition, chemical properties, and stability of blends made from a variety of common edible vegetable oils with linseed oil. 


\subsection{Material.}

\subsubsection{Procurement of oils/fats.}

Common oils were collected from Sadar Bazar, Ambala Cantt., India. Oils included coconut, olive, soybean, mustard, linseed, and sunflower oil. All oils/fats were freshly extracted from the nuts and seed using the cold-press process.

\subsubsection{Reagents and chemicals.}

The mixture of fatty acid standards was procured from Supelco (Sigma Aldrich, Germany). The other chemicals and reagents used for fatty acids methyl esters (FAME) synthesis were of HPLC grade, and the remaining chemicals were of AR grade purchased from Central Drug House (CDH), New Delhi, India.

\subsection{Blending of oils/fats.}

Generally, blending refers to the mixing of different types of substances having a similar composition to make a product of desirable quality. For blending different edible oils such as mustard oil, olive oil, coconut oil, sunflower oil, and soybean oils were taken in different ratios with linseed oil (Table 1). The mixtures of oils/fats were blended in conical flasks using a magnetic stirrer at a constant temperature of $40^{\circ} \mathrm{C}$ for about one hour.

Table 1.Different oils/fats blend with linseed oil in different proportions.

\begin{tabular}{|c|c|c|c|c|c|}
\hline Oil & 1 & 2 & 3 & 4 & 5 \\
\hline \multirow{7}{*}{ Blends (v/v) } & $20: 80$ & $20: 80$ & $20: 80$ & $20: 80$ & $20: 80$ \\
\hline & $30: 70$ & $30: 70$ & $30: 70$ & $30: 70$ & $30: 70$ \\
\hline & $40: 60$ & $40: 60$ & $40: 60$ & $40: 60$ & $40: 60$ \\
\hline & $50: 50$ & $50: 50$ & $50: 50$ & $50: 50$ & $50: 50$ \\
\hline & $60: 40$ & $60: 40$ & $60: 40$ & $60: 40$ & $60: 40$ \\
\hline & $70: 30$ & $70: 30$ & $70: 30$ & $70: 30$ & $70: 30$ \\
\hline & $80: 20$ & $80: 20$ & $80: 20$ & $80: 20$ & $80: 20$ \\
\hline
\end{tabular}

1-LO:MO, 2-LO: SBO, 3-LO: SFO, 4-LO:CO, 5-LO: OO

LO: Linseed oil; MO: Mustard oil; SBO: Soybean oil; SFO: Sunflower oil; CO: Coconut oil; OO: Olive oil

\subsection{Chemical properties of oils/fats.}

Chemical properties (Acid value, peroxide value, and iodine value) of all the oils were studied before and after blending as per standard procedures given by FSSAI, 2011 regarding fats and oils [4].

\subsection{Fatty acid composition.}

FAME synthesis was carried out using the method of direct FAME synthesis by O'Fallon et al. (2007) [19]. Forty microliter oil sample was taken in a screw cap Pyrex culture tubes added with $0.7 \mathrm{ml}$ of $10 \mathrm{~N} \mathrm{KOH}$ followed by $5.3 \mathrm{ml}$ of methanol. Tubes were placed in a hot water bath at $55{ }^{\circ} \mathrm{C}$ for 1.5 hours with vigorous handshaking for 5 seconds after every 20minute intervals to dissolve and hydrolyze the sample. Tubes were cooled at room temperature, and $0.58 \mathrm{ml}$ of $24 \mathrm{~N} \mathrm{H}_{2} \mathrm{SO}_{4}$ was added and mixed properly. After the formation of $\mathrm{K}_{2} \mathrm{SO}_{4}$ precipitates, tubes were again placed in a water bath at $55{ }^{\circ} \mathrm{C}$ for 1.5 hours with handshaking for 5 seconds each after 20-minute intervals as performed earlier. After that, these tubes were cooled at room temperature, and $3 \mathrm{ml}$ hexane was added and vortexed for 5 minutes. Thereafter, 
tubes were centrifuged for 5 minutes, and the top hexane layer containing FAMEs were placed in $\mathrm{GC}$ vials and stored at $-20^{\circ} \mathrm{C}$ until GC analysis [19].

The prepared FAMEs of individual and blended oil samples were injected into the Perkin Elmer, Clarus 680 gas chromatograph equipped with an FID detector. Zebron ${ }^{\mathrm{TM}} \mathrm{ZB}-$ WAXplus $^{\mathrm{TM}}$, Capillary Column $(60 \mathrm{~m}$ x $0.32 \mathrm{~mm}$ x $0.25 \mu \mathrm{m})$, packed with Polyethylene Glycol (PEG) as stationary phase, was used for the separation and identification of fatty acids using the following instrumental conditions; injector temperature: $230{ }^{\circ} \mathrm{C}$; detector temperature: 240 ${ }^{\circ} \mathrm{C}$; carrier gas: nitrogen @ 1 $1 \mathrm{ml} / \mathrm{min}$ in splitless mode; oven program; starting at $100{ }^{\circ} \mathrm{C}$ (held for 3 minutes) to $150{ }^{\circ} \mathrm{C} @ 7{ }^{\circ} \mathrm{C}$ (held for 2 minutes) to $230{ }^{\circ} \mathrm{C} @ 7{ }^{\circ} \mathrm{C}$ (held for 5 minutes); injector volume: $1 \mu \mathrm{L}$. The obtained fatty acids were quantified by calculating area percentage comparing Supelco 37 FAME mixture as the reference standard.

\subsection{Storage study.}

All blended samples were stored at room temperature and analyzed for their quality as per the standards procedures given by FSSAI, 2011 [4]. Storage study was carried out for up to four months, and samples were drawn for analysis after one month of interval.

\subsection{Data analysis.}

Each sample of the individual as well as a mixture of oils/fats (fresh and stored), was analyzed for chemical parameters (acid value, peroxide value, and iodine value), and one-way analysis of variance (ANOVA) was calculated and significant difference between the values was observed. All experiments were conducted in triplicates. Mean values with no common superscript are significantly different $(\mathrm{p}<0.05)$, according to Duncan's multiple range test. The data analysis was performed with the help of SPSS version 23 . Whereas the fatty acid profile was taken in triplicates, and the mean values were reported with \pm standard deviation.

\section{Results and Discussion}

The present investigation was carried out to prepare and study the storage stability of various oil blends, where linseed oil was taken as base oil, while other oils were added in different ratios.

\subsection{Chemical properties of individual edible oils.}

As depicted in Table 2, acid values of linseed oil (1.07 \pm 0.34$)$ and sunflower oil $(1.06 \pm 0.07)$ were significantly higher than other oils, while the lowest value was observed in coconut oil $(0.40 \pm 0.34 \%)$. The peroxide value of mustard oil $\left(3.10 \pm 0.42 \mathrm{meqO}_{2} / \mathrm{kg}\right)$ was the highest among all oils. Because of the higher concentration of PUFA, iodine value was observed significantly high in linseed oil (176.32 27.04$)$. At the same time, coconut oil showed the lowest iodine value $(10.14 \pm 1.12)$ as it contains more of an SFA. The results obtained were found in accordance with the value given by different researchers for $\mathrm{LO}, \mathrm{CO}, \mathrm{SBO}, \mathrm{OO}$, and MO [20-25].

\subsection{Chemical properties of oil blends.}

Analysis of blended samples (Table 3) showed that acid value existed under the standard limit ( $\leq 2$ for all edible oils) as prescribed by FSSR, 2011. Similarly, the peroxide 
value (Table 4) was also found under the prescribed standard limit $(\leq 10 \mathrm{meqO} / \mathrm{kg})$. On the other hand, iodine value (Table 5) was affected significantly due to the specific properties and fatty acid profile of individual oils, i.e., linseed oil has the highest and coconut oil has the lowest iodine value. Therefore, when such types of oils were blended in different proportions, their degree of unsaturation, along with iodine value, was affected accordingly.

Table 2.Chemical properties of individual edible oils.

\begin{tabular}{l|l|l|l} 
Sample & Acid Value & Peroxide Value & Iodine Value \\
\hline Linseed Oil & $1.07 \pm 0.34^{\mathrm{f}}$ & $1.40 \pm 0.20^{\mathrm{e}}$ & $176.32 \pm 7.04^{\mathrm{f}}$ \\
\hline Coconut Oil & $0.40 \pm 0.34^{\mathrm{a}}$ & $0.61 \pm 0.64^{\mathrm{a}}$ & $010.14 \pm 1.12^{\mathrm{a}}$ \\
\hline Soybean Oil & $0.80 \pm 0.11^{\mathrm{c}}$ & $1.06 \pm 1.86^{\mathrm{d}}$ & $120.38 \pm 6.42^{\mathrm{d}}$ \\
\hline Sunflower Oil & $01.06 \pm 0.07^{\mathrm{e}}$ & $1.02 \pm 0.31^{\mathrm{c}}$ & $129.22 \pm 4.87^{\mathrm{e}}$ \\
\hline Olive Oil & $00.49 \pm 0.10^{\mathrm{b}}$ & $0.81 \pm 0.25^{\mathrm{b}}$ & $086.01 \pm 3.06^{\mathrm{b}}$ \\
\hline Mustard Oil & $01.00 \pm 0.17^{\mathrm{d}}$ & $3.10 \pm 0.42^{\mathrm{f}}$ & $099.52 \pm 4.33^{\mathrm{c}}$
\end{tabular}

Values are means of triplicate \pm standard deviation

Within a column with the same lowercase letter are not significantly different at $p<0.05$

Table 3.Acid value of oils/fats blends.

\begin{tabular}{l|l|l|l|l|l} 
& \multicolumn{6}{|l}{ Acid Value of Different Blends } \\
\hline Ratios & $\mathbf{1}$ & $\mathbf{2}$ & $\mathbf{3}$ & $\mathbf{4}$ & $\mathbf{5}$ \\
\hline $\mathbf{2 0 : 8 0}$ & $1.12 \pm 0.08^{\mathrm{ij}}$ & $1.06 \pm 0.11^{\mathrm{g}}$ & $1.12 \pm 0.10^{\mathrm{ij}}$ & $0.37 \pm 0.02^{\mathrm{a}}$ & $0.55 \pm 0.04^{\mathrm{ef}}$ \\
\hline $\mathbf{3 0 : 7 0}$ & $1.10 \pm 0.11^{\mathrm{i}}$ & $1.10 \pm 0.20^{\mathrm{i}}$ & $1.10 \pm 0.09^{\mathrm{i}}$ & $0.39 \pm 0.03^{\mathrm{b}}$ & $0.55 \pm 0.08^{\mathrm{ef}}$ \\
\hline $\mathbf{4 0 : 6 0}$ & $1.12 \pm 0.10^{\mathrm{ij}}$ & $1.12 \pm 0.18^{\mathrm{j}}$ & $1.07 \pm 0.10^{\mathrm{g}}$ & $0.38 \pm 0.07^{\mathrm{a}}$ & $0.54 \pm 0.03^{\mathrm{e}}$ \\
\hline $\mathbf{5 0 : 5 0}$ & $1.12 \pm 0.90^{\mathrm{ij}}$ & $1.12 \pm 0.10^{\mathrm{ij}}$ & $1.12 \pm 0.12^{\mathrm{j}}$ & $0.40 \pm 0.09^{\mathrm{b}}$ & $0.55 \pm 0.09^{\mathrm{ef}}$ \\
\hline $\mathbf{6 0 : 4 0}$ & $1.18 \pm 0.09^{\mathrm{k}}$ & $1.10 \pm 0.07^{\mathrm{i}}$ & $1.10 \pm 0.08^{\mathrm{i}}$ & $0.41 \pm 0.05^{\mathrm{c}}$ & $0.56 \pm 0.04^{\mathrm{ef}}$ \\
\hline $\mathbf{7 0 : 3 0}$ & $1.12 \pm 0.10^{\mathrm{j}}$ & $1.08 \pm 0.11^{\mathrm{h}}$ & $1.10 \pm 0.12^{\mathrm{i}}$ & $0.40 \pm 0.03^{\mathrm{b}}$ & $0.55 \pm 0.07^{\mathrm{ef}}$ \\
\hline $\mathbf{8 0 : 2 0}$ & $1.12 \pm 0.11^{\mathrm{j}}$ & $1.12 \pm 0.10^{\mathrm{j}}$ & $1.12 \pm 0.09^{\mathrm{j}}$ & $0.41 \pm 0.04^{\mathrm{bc}}$ & $0.56 \pm 0.08^{\mathrm{ef}}$
\end{tabular}

Values are means of triplicate \pm standard deviation

Within a raw with the same lowercase letter are not significantly different at $p<0.05$

Table 4.Peroxide value of oils/fats blends.

\begin{tabular}{l|c|c|c|c|c} 
& \multicolumn{5}{|c}{ Peroxide Value of Different Blends } \\
\hline Ratios & $\mathbf{1}$ & $\mathbf{2}$ & $\mathbf{3}$ & $\mathbf{4}$ & $\mathbf{5}$ \\
\hline $\mathbf{2 0 : 8 0}$ & $2.40 \pm 0.88^{\mathrm{i}}$ & $1.20 \pm 0.34^{\mathrm{d}}$ & $1.00 \pm 0.78^{\mathrm{c}}$ & $1.11 \pm 0.32^{\mathrm{c}}$ & $1.60 \pm 0.75^{\mathrm{e}}$ \\
\hline $\mathbf{3 0 : 7 0}$ & $2.20 \pm 0.95^{\mathrm{h}}$ & $1.12 \pm 0.16^{\mathrm{c}}$ & $0.82 \pm 0.91^{\mathrm{a}}$ & $0.81 \pm 0.21^{\mathrm{a}}$ & $0.80 \pm 0.09^{\mathrm{a}}$ \\
\hline $\mathbf{4 0 : 6 0}$ & $1.80 \pm 0.38^{\mathrm{f}}$ & $0.81 \pm 0.27^{\mathrm{a}}$ & $1.00 \pm 1.03^{\mathrm{b}}$ & $1.19 \pm 0.68^{\mathrm{d}}$ & $1.11 \pm 0.62^{\mathrm{c}}$ \\
\hline $\mathbf{5 0 : 5 0}$ & $1.60 \pm 0.90^{\mathrm{e}}$ & $0.81 \pm 0.60^{\mathrm{a}}$ & $0.81 \pm 0.88^{\mathrm{a}}$ & $1.10 \pm 0.78^{\mathrm{c}}$ & $1.11 \pm 0.69^{\mathrm{c}}$ \\
\hline $\mathbf{6 0 : 4 0}$ & $1.81 \pm 0.66^{\mathrm{f}}$ & $1.20 \pm 0.55^{\mathrm{d}}$ & $1.21 \pm 0.82^{\mathrm{d}}$ & $0.81 \pm 0.06^{\mathrm{a}}$ & $1.12 \pm 0.90^{\mathrm{c}}$ \\
\hline $\mathbf{7 0 : 3 0}$ & $2.20 \pm 0.10^{\mathrm{h}}$ & $1.00 \pm 0.42^{\mathrm{b}}$ & $0.81 \pm 0.40^{\mathrm{a}}$ & $0.82 \pm 0.10^{\mathrm{a}}$ & $1.20 \pm 0.10^{\mathrm{d}}$ \\
\hline $\mathbf{8 0 : 2 0}$ & $2.11 \pm 0.09^{\mathrm{g}}$ & $1.00 \pm 0.48^{\mathrm{b}}$ & $1.00 \pm 0.95^{\mathrm{b}}$ & $1.11 \pm 0.10^{\mathrm{c}}$ & $1.12 \pm 0.10^{\mathrm{c}}$
\end{tabular}

Values are means of triplicate \pm standard deviation

Within a raw with the same lowercase letter are not significantly different at $\mathrm{p}<0.05$

Table 5.Iodine value of oils/fats blends.

\begin{tabular}{l|c|c|c|c|c} 
& \multicolumn{5}{|c}{ Iodine Value of Different Blends } \\
\hline Ratios & $\mathbf{1}$ & $\mathbf{2}$ & $\mathbf{3}$ & $\mathbf{4}$ & $\mathbf{5}$ \\
\hline $\mathbf{2 0 : 8 0}$ & $110.23 \pm 4.50^{\mathrm{i}}$ & $119.25 \pm 4.38^{\mathrm{h}}$ & $120.45 \pm 3.03^{\mathrm{p}}$ & $061.69 \pm 2.24^{\mathrm{a}}$ & $111.23 \pm 3.72^{\mathrm{j}}$ \\
\hline $\mathbf{3 0 : 7 0}$ & $114.93 \pm 5.11^{1}$ & $121.26 \pm 6.07^{\mathrm{q}}$ & $124.33 \pm 3.60^{\mathrm{r}}$ & $068.90 \pm 3.49^{\mathrm{b}}$ & $113.36 \pm 4.21^{\mathrm{k}}$ \\
\hline $\mathbf{4 0 : 6 0}$ & $117.88 \pm 4.15^{\mathrm{n}}$ & $124.65 \pm 3.94^{\mathrm{rs}}$ & $129.78 \pm 2.90^{\mathrm{w}}$ & $076.55 \pm 4.72^{\mathrm{c}}$ & $116.82 \pm 3.27^{\mathrm{m}}$ \\
\hline $\mathbf{5 0 : 5 0}$ & $125.87 \pm 3.55^{\mathrm{t}}$ & $130.92 \pm 4.34^{\mathrm{x}}$ & $138.45 \pm 3.05^{\mathrm{a} 1}$ & $082.38 \pm 3.47^{\mathrm{d}}$ & $118.57 \pm 5.45^{\mathrm{o}}$ \\
\hline $\mathbf{6 0 : 4 0}$ & $129.63 \pm 5.44^{\mathrm{vw}}$ & $139.28 \pm 5.51^{\mathrm{a} 2}$ & $140.35 \pm 4.92^{\mathrm{a} 3}$ & $086.88 \pm 2.48^{\mathrm{e}}$ & $124.85 \pm 3.09^{\mathrm{s}}$ \\
\hline $\mathbf{7 0 : 3 0}$ & $132.47 \pm 5.32^{\mathrm{y}}$ & $143.28 \pm 5.10^{\mathrm{a}}$ & $141.92 \pm 4.50^{\mathrm{a} 4}$ & $090.45 \pm 3.25^{\mathrm{f}}$ & $127.56 \pm 5.18^{\mathrm{u}}$ \\
\hline $80: 20$ & $135.93 \pm 5.12^{\mathrm{z}}$ & $150.57 \pm 5.21^{\mathrm{a} 7}$ & $146.47 \pm 3.11^{\mathrm{a} 6}$ & $093.93 \pm 5.78^{\mathrm{g}}$ & $129.23 \pm 3.24^{\mathrm{v}}$
\end{tabular}

Values are means of triplicate \pm standard deviation

Within a raw with the same lowercase letter are not significantly different at $\mathrm{p}<0.05$

\subsection{Fatty acid composition of oils/fats before and after blending.}

The FAMEs of individual, as well as blended oil samples, were analyzed for the fatty acid profile using gas chromatography. The results obtained for fresh oils were illustrated in 
Table 6, and, Figure 1 and 2 reveals the percentage and ratios of SFA, MUFA, and PUFA in blended samples.

Table 6.Fatty acid composition of common edible oils.

\begin{tabular}{l|c|c|c|c|c|c}
\multirow{2}{*}{ Fatty Acids } & \multicolumn{7}{|c}{ Oil Samples } \\
\cline { 2 - 7 } & Linseed & Coconut & Mustard & Soybean & Sunflower & Olive \\
\hline C8:0 & - & $05.85 \pm 1.03$ & - & - & - & - \\
\hline C10:0 & - & $04.86 \pm 0.76$ & - & - & - & - \\
\hline C12:0 & $02.13 \pm 0.34$ & $47.97 \pm 4.21$ & - & - & - & - \\
\hline C14:0 & $00.99 \pm 0.04$ & $19.35 \pm 2.33$ & - & - & - & $14.06 \pm 2.21$ \\
\hline C16:0 & $05.79 \pm 0.76$ & $08.80 \pm 1.63$ & $02.62 \pm 0.21$ & $06.55 \pm 1.31$ & $06.97 \pm 0.86$ & $01.11 \pm 0.32$ \\
\hline C16:1 & - & - & - & $00.07 \pm 0.00$ & - & $03.02 \pm 0.54$ \\
\hline C18:0 & $04.69 \pm 0.47$ & $02.49 \pm 0.22$ & $01.24 \pm 0.06$ & $03.29 \pm 0.43$ & $03.28 \pm 0.51$ & $71.15 \pm 5.74$ \\
\hline C18:1 & $19.29 \pm 1.23$ & $08.11 \pm 1.243$ & $11.70 \pm 1.12$ & $26.90 \pm 3.54$ & $26.21 \pm 2.34$ & $09.59 \pm 1.76$ \\
\hline C18:2 & $13.36 \pm 1.43$ & $02.54 \pm 0.16$ & $16.05 \pm 1.65$ & $59.23 \pm 4.38$ & $62.67 \pm 4.48$ & $00.99 \pm 0.24$ \\
\hline C18:3 & $51.95 \pm 3.72$ & - & $12.47 \pm 2.36$ & $03.00 \pm 0.38$ & $00.29 \pm 0.01$ & - \\
\hline C20:0 & $00.21 \pm 0.02$ & - & $04.89 \pm 0.52$ & $00.12 \pm 0.00$ & $00.57 \pm 0.01$ & - \\
\hline C20:1 & - & - & $01.05 \pm 0.04$ & $00.07 \pm 0.01$ & - & - \\
\hline C20:2 & - & - & $00.63 \pm 0.02$ & - & - & - \\
\hline C22:0 & $01.56 \pm 0.22$ & - & $01.11 \pm 0.24$ & $00.62 \pm 0.02$ & - & - \\
\hline C22:1 & - & - & $44.06 \pm 2.43$ & - & - & - \\
\hline C23:0 & - & - & - & $00.17 \pm 0.00$ & - & - \\
\hline C24:0 & - & - & $01.18 \pm 0.34$ & - & - & - \\
\hline C24:1 & - & - & $02.04 \pm 0.41$ & - & - & $\mathbf{1 0 . 0 8}$ \\
\hline SFA & $\mathbf{1 5 . 3 7}$ & $\mathbf{8 9 . 3 2}$ & $\mathbf{1 1 . 0 4}$ & $\mathbf{1 0 . 7 5}$ & $\mathbf{1 0 . 8 2}$ & $\mathbf{1 7 . 0 8}$ \\
\hline MUFA & $\mathbf{1 9 . 2 9}$ & $\mathbf{0 8 . 1 1}$ & $\mathbf{5 8 . 8 5}$ & $\mathbf{2 7 . 0 4}$ & $\mathbf{2 6 . 2 1}$ & $\mathbf{7 2 . 2 6}$ \\
\hline PUFA & $\mathbf{6 5 . 3 1}$ & $\mathbf{0 2 . 5 4}$ & $\mathbf{2 9 . 1 5}$ & $\mathbf{6 2 . 2 3}$ & $\mathbf{6 2 . 9 6}$ & $\mathbf{1 0 . 5 8}$ \\
\hline SFA:MUFA:PUFA & $\mathbf{1 : 1 . 2 : 4 . 3}$ & $\mathbf{3 5 . 1 : 3 . 1 : 1}$ & $\mathbf{1 : 5 . 3 : 2 . 6}$ & $\mathbf{1 : 2 . 5 : 5 . 7}$ & $\mathbf{1 : 2 . 4 : 5 . 8}$ & $\mathbf{1 . 6 : 6 . 8 : 1}$
\end{tabular}

Values are means of triplicate \pm standard deviation

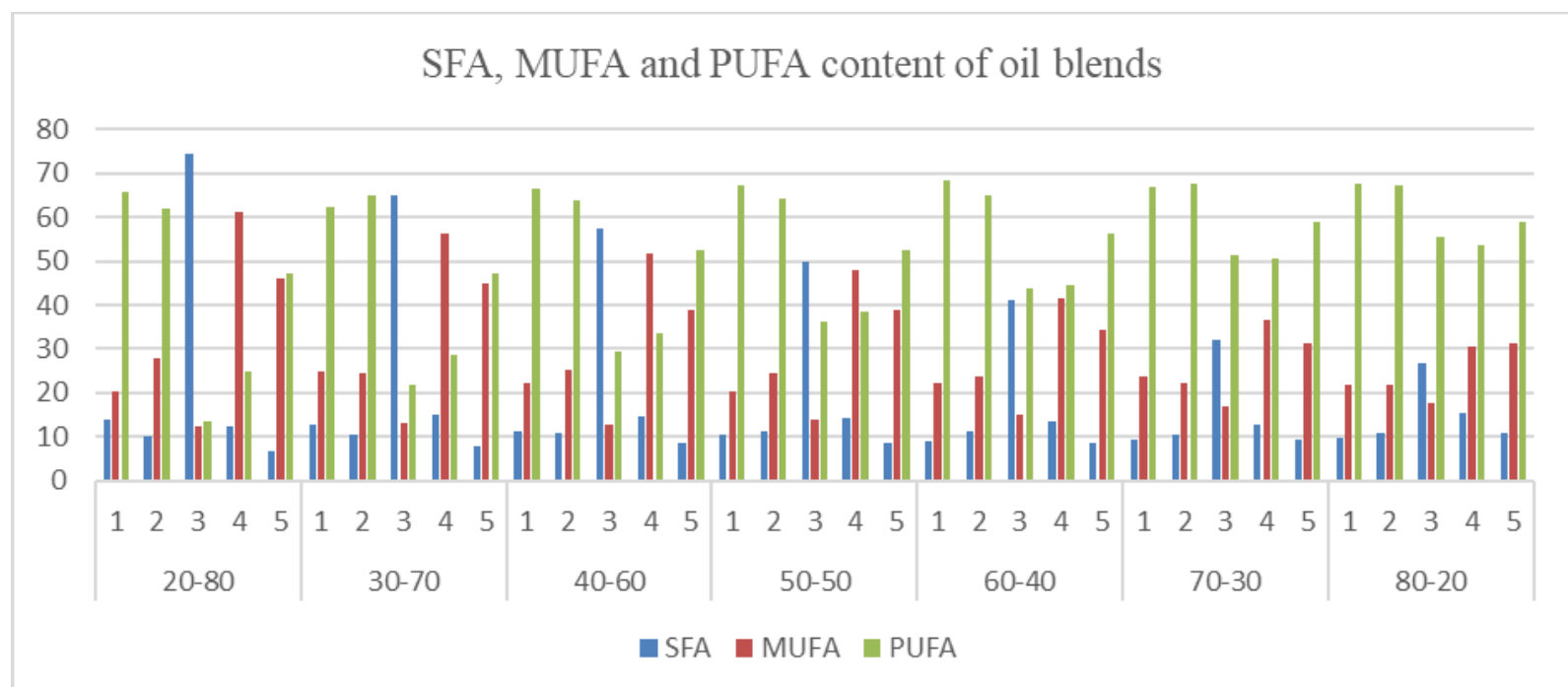

1-Linseed and Soybean oil blend, 2-Linseed and Sunflower oil blend, 3-Linseed and Coconut oil blend, 4-Linseed and Olive oil blend, 5Linseed and Mustard oil blend

Figure 1. Percentage of SFA, MUFA, and PUFA in blended samples.

3.3.1. Fatty acid profile of individual oils/fats.

As revealed from the fatty acid profile of individual vegetable oils (Table 6), LO contained a high amount of ALA (51.95 \pm 3.72$)$ followed by oleic acid (19.29 \pm 1.23$)$ and linoleic acid (13.61 \pm 1.43$)$. While coconut oil showed a very different fatty acid profile as it contained more amount of medium-chain fatty acids dominated by lauric acid (47.97 \pm 4.21$)$ along with a significant amount of myristic acid (19.35 \pm 2.33$)$ and have a very low level of MUFA and PUFA. 


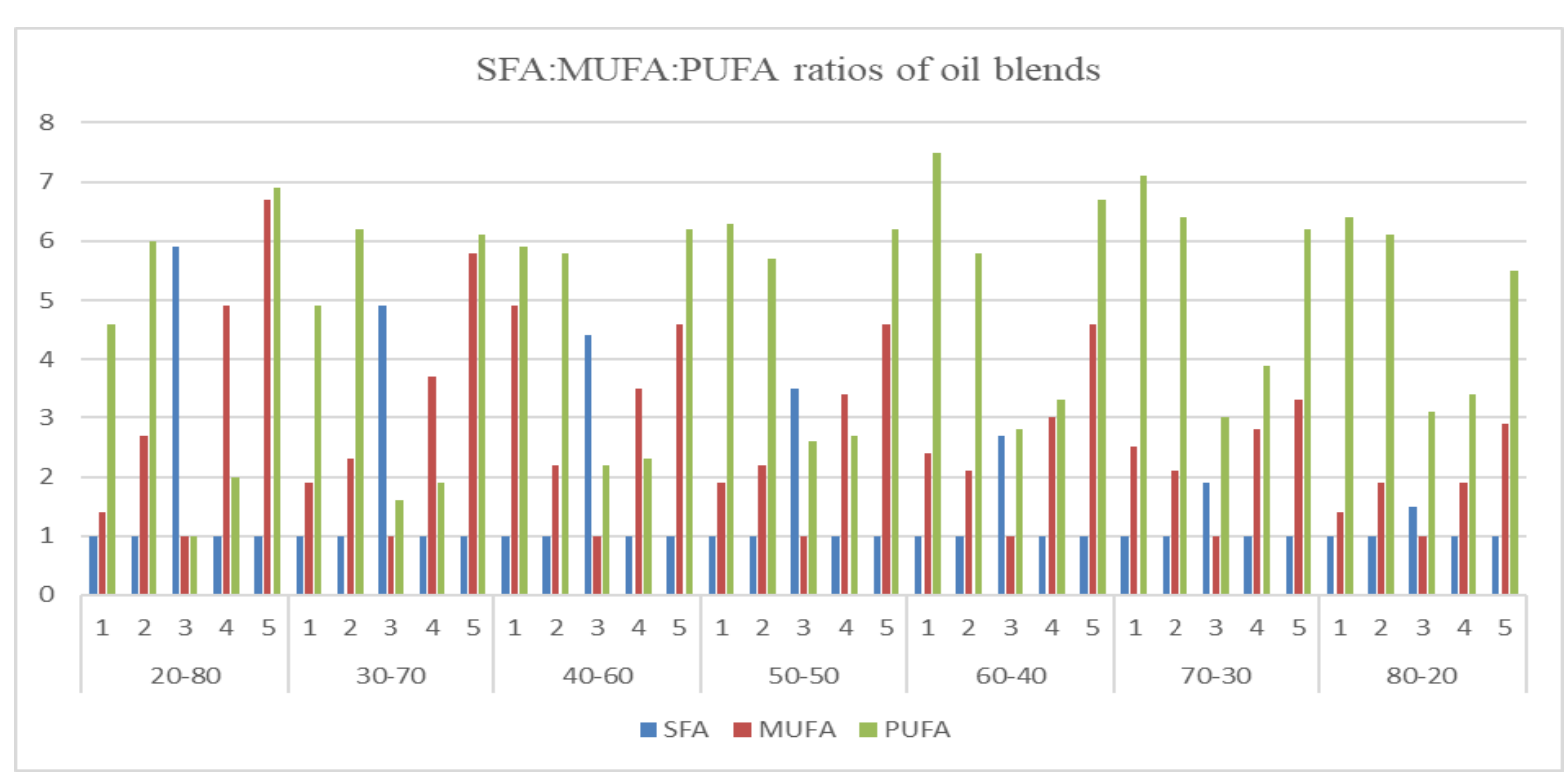

1-Linseed and Soybean oil blend, 2- Linseed and Sunflower oil blend, 3-Linseed and Coconut oil blend, 4-Linseed and Olive oil blend, 5Linseed and Mustard oil blend

Figure 2. Ratio of SFA, MUFA, and PUFA content in blended samples.

Mustard oil has a unique composition because of high erucic acid (44.06 \pm 2.43$)$ content; other fatty acids such as oleic (11.70 \pm 1.12$)$, linoleic $(16.05 \pm 1.65)$, and linolenic acid $(12.47 \pm 2.36)$ were also present in significant amounts. In the fatty acid composition of soybean oil, linoleic acid $(59.23 \pm 4.38)$ was found predominating, which contributed to more than half

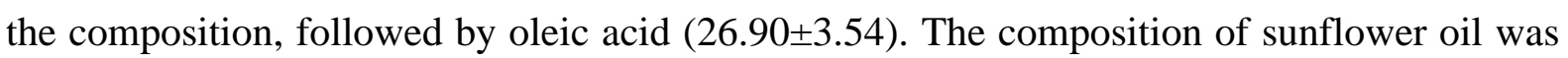
found close to soybean oil as it also contained a high amount of linoleic acid (62.67 \pm 4.48$)$, and the second dominating fatty acid was oleic acid (26.21 \pm 2.34$)$. Olive oil is well known for its better frying properties because of its high oleic acid content. The results showed the maximum content of oleic $(71.15 \pm 5.74)$ along with a significant amount of palmitic (14.06 \pm 2.21$)$ and linoleic acid (09.59 \pm 1.76$)$. A study conducted by various researchers reported a similar pattern of fatty acids profile of common edible vegetable oils [1-2, 5, 7, 10, 20, 21].

The ratio of total SFA:MUFA:PUFA in different oils greatly affects the stability, digestibility, and quality of the respective oil [26]. It was observed that the calculated value of SFA:MUFA:PUFA of each oil was significantly different from each other and were specific according to their respective composition. Linseed oil contained the maximum amount of PUFA (65.31) amongst selected oils samples, and the ratio of SFA:MUFA:PUFA was 1:1.2:4.3. Whereas coconut oil showed an opposite pattern in ratio, i.e., 35.1:3.1:1. In the case of mustard, soybean, and sunflower oil, the ratios were 1:5.3:2.6; 1:2.5:5.7, and 1:2.4:5.8, respectively. On the other hand, olive oil has the highest MUFA content, showed a ratio of SFA:MUFA:PUFA was 1.6:6.8:1. It was concluded from the compositional study that no individual oil has a composition that meets the recommendation given by WHO, 2008 [18]. Therefore, the blending of selected oils was performed to adjust composition in line with recommended levels of SFA, MUFA, and PUFA.

\subsubsection{Fatty acid composition of oil blends.}

After analyzing the fatty acid composition of individual oil samples, the respective oil blends were also studied for their total SFA, MUFA, and PUFA content, which is shown in Figures 1 and 2. 
As revealed in Figure 1, PUFA content of blends increased with an increasing proportion of linseed oil, while SFA and MUFA content varies according to the unique fatty acid composition of individual oil. In LO and SBO blend, the proportion of 20:80 showed SFA:MUFA:PUFA content in a ratio of 1:1.4:4.6, while the blend of 80:20 showed a ratio of 1:1.4:6.4 in which the former was observed close to recommended intake [18]. In a blend of LO and SFO, MUFA and PUFA content were noticed higher than the recommended level, as both oils have high PUFA content (linoleic and linolenic acids). In this set of blends, none of the blends were found suitable as per their fatty acid composition.

The blend of LO and $\mathrm{CO}$ was an interesting combination because both oils have opposite properties. LO is rich in PUFA content; on the other hand, CO contained a high amount of SFA. CO shows SFA:MUFA:PUFA ratio as 35.1:3.1:1, while LO has 1:1.2:4.3. When both the oils were blended, the fatty acid composition was affected drastically. In blend of 20:80, SFA:MUFA:PUFA ratio was 5.9:1:1 followed by 4.9:1:1.6, 4.4:1:2.2, 3.5:1:2.6, 2.7:1:2.8, 1.9:1:3 and 1.5:1:3.1 respectively for remaining blends (Figure 2). As mentioned above, a blend of 80:20 showed SFA:MUFA:PUFA content close to the recommended level. LO and $\mathrm{OO}$ blends also showed good compositional balance. Olive oil has a high smoke point, so it can be a good choice as a frying medium, but the economic value of this oil is comparatively high. Therefore, the blending of OO with any low-priced oil could be a better economical option. Blend of LO and OO in ratio 80:20 showed SFA:MUFA:PUFA content in ratio 1:1.9:3.4, which was found close to the suggested ratio. In a blend of LO and MO, none of the proportions were observed to be near the recommended level.

\subsection{Storage study of oil blends.}

The chemical properties of different oil blends stored at room temperature were studied up to 120 days. The samples were drawn to check their quality after one-month intervals. Acid, peroxide, and iodine values are depicted in Figure 3 to 5.

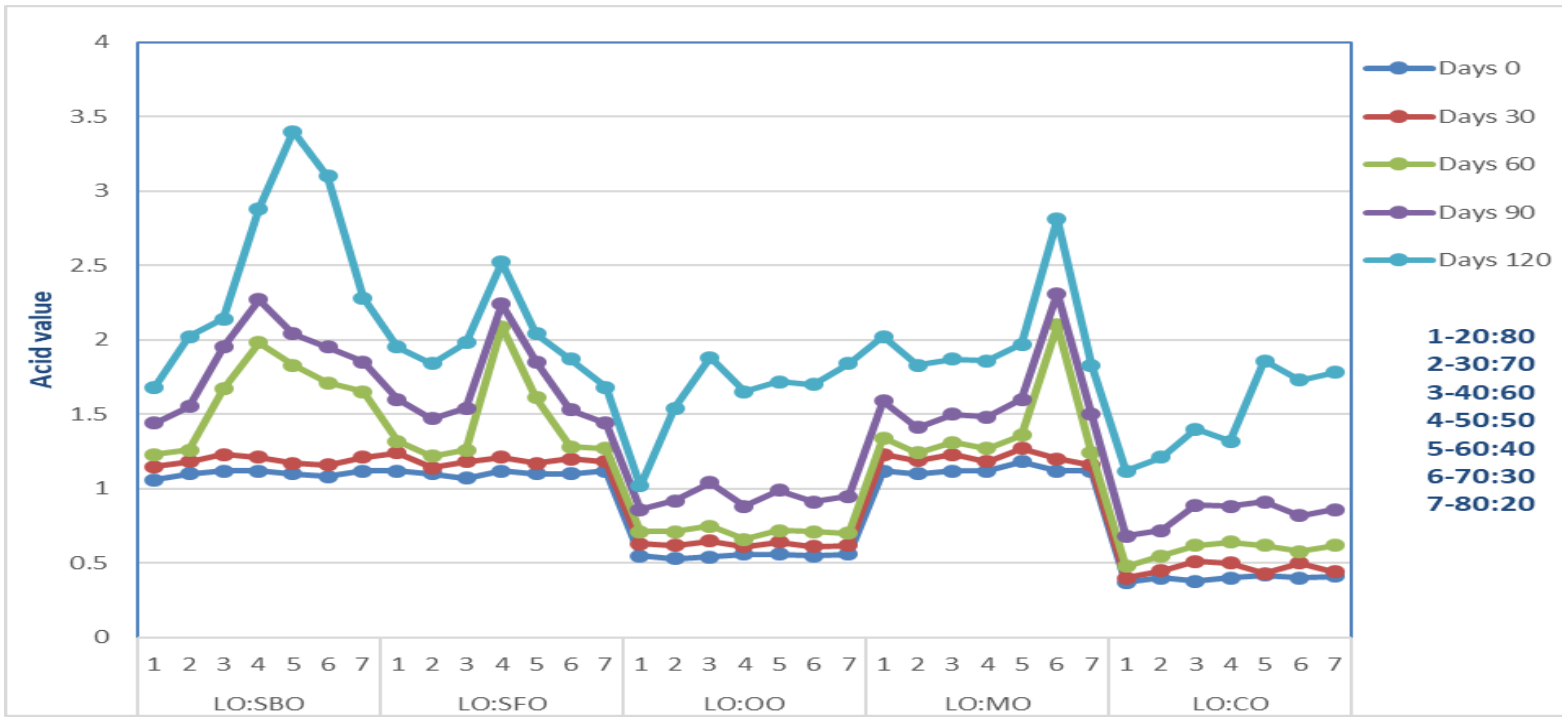

Figure 3. Acid value of oil blends at different storage times.

The acid value is mostly used to check the quality of oils and fats. The limits for acid value for individual oils/fats are already mentioned by FSSAI, 2011 [4]. As presented in Figure 3 , acid values of different blends at zero days ranged from 1.06 to $1.12,1.07$ to $1.12,0.53$ to 
0.56, 1.10 to 1.18 , and 0.37 to 0.42 for LO:SBO, LO:SFO, LO:OO, LO:MO and LO:CO blends, respectively and were observed under the standard limit.

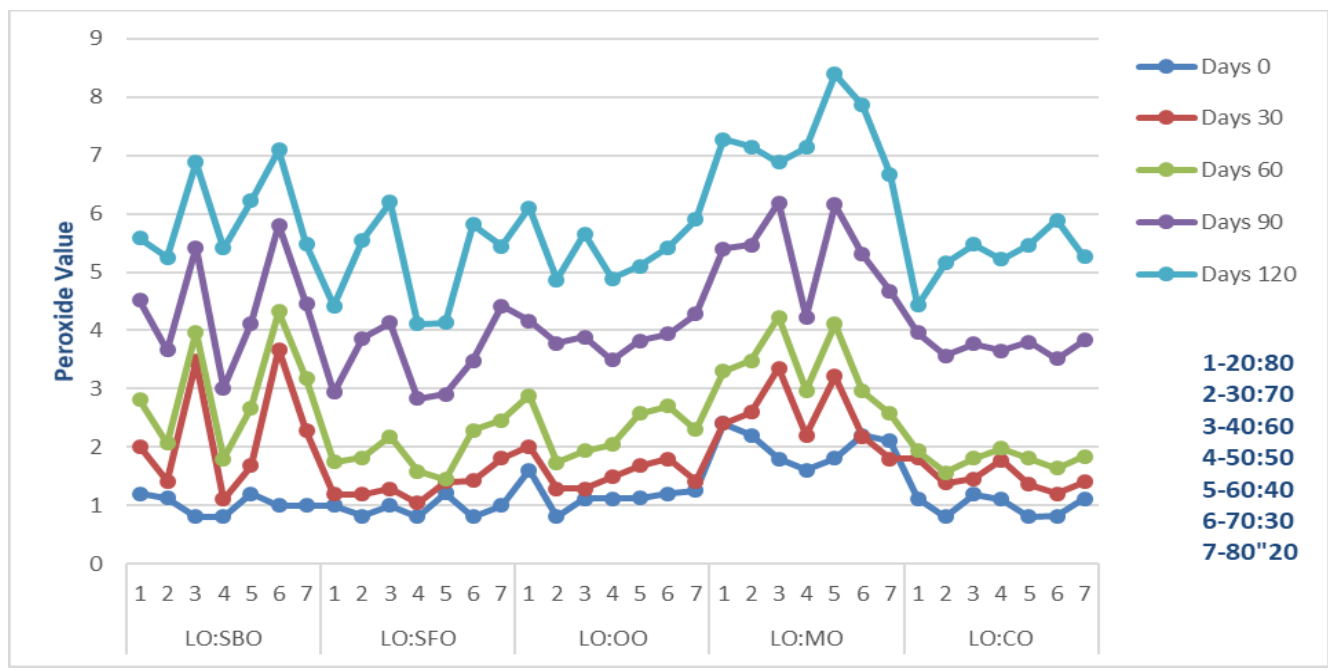

Figure 4. Peroxide value of oil blends at different storage times.

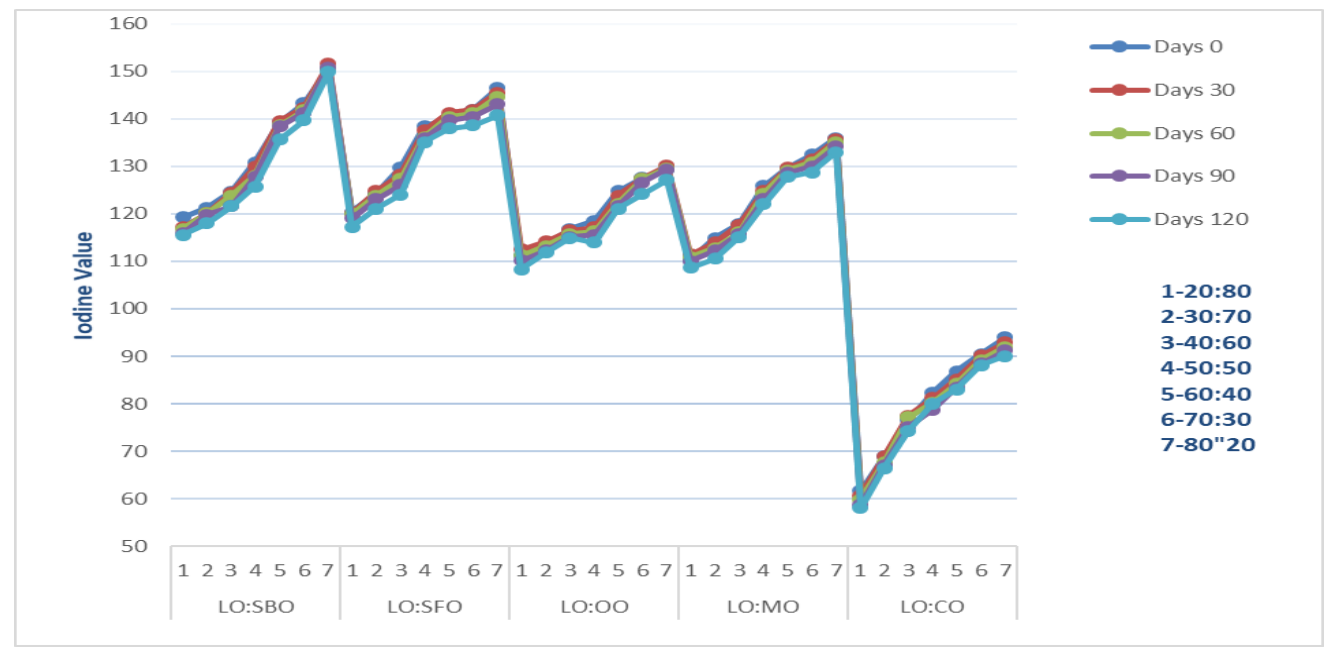

Figure 5. Iodine value of oil blends at different storage time.

With an increase in the storage period, the quality of oils was affected due to an increase in acid value [27]. Up to 60 days of storage, all the blended samples were found stable except blends of LO:SFO (50:50) and LO:MO (70:30). After 90 days, 0.2 to 0.3 percent increase was observed in acid value, and some of the samples such as LO:SBO (50:50), LO:SBO (60:40), LO:SFO (50:50), LO:MO (70:30) exceeded the standard limit possibly because of high concentration of PUFA in respective oil blends. Further, up to 120 days of storage, 8 samples out of 35 were degraded, and the maximum acid value was recorded in LO:SBO (60:40) blend. Results from different studies [28-29] revealed a similar pattern of increase in acid value, which is greatly affected by storage conditions and period.

Peroxide value represents the concentration of peroxides and hydroperoxides generated due to lipid oxidation. It is an important parameter to determine the oxidative stability of oils and fats [30]. FSSAI, 2011 has prescribed the standard for peroxide value, which should be below $10 \mathrm{meq} / \mathrm{Kg}$ as the rancidity tolerance limit of fresh oils [4]. A noticeable rancid taste will appear as the value rises above $20 \mathrm{meq} / \mathrm{Kg}$ (this is the standard limit for most industries). A continuous increase in peroxide value was observed with an increase in the storage period in all the samples (Figure 4). The samples were found fresh at the initial stage and up to 120 days 
of storage. Hence, no rancid flavor was observed even in a single sample throughout the storage time.

Iodine value is used to check fat stability and resistance to oxidation by measuring the degree of unsaturation. It is significantly affected by storage conditions and time because of the formation of peroxides and hydroperoxides [31]. In the present investigation, it was observed that the iodine value decreased slightly with an increase in storage time. This may be due to the susceptibility of unsaturated bonds to oxidation (Figure 5). The maximum decrease (4.4 percent) in iodine value was observed in LO:SFO (40:60) blend, as the respective oils, have a high degree of unsaturation. While, in the remaining samples, a significant decrease was observed, compared to a lesser extent.

\section{Conclusions}

The present study was designed to improve the quality of oils using a physical blending process, as no single conventional oil has the ideal fatty acid ratio. Various blends were prepared and studied for their quality. As per the recommended level of SFA:MUFA:PUFA, all samples were examined, and blends of LO:SBO (20:80), LO:CO (80:20), and LO:OO $(80: 20)$ were found close to the recommended intake $(1: 1.5: 1)$. Keeping the quality of maximum samples was also improved and observed well, up to 120 days of storage at room temperature. These blends were nutritionally better as linseed oil contained $\omega$-3-fatty acid, which is well known for its anti-obesity, anti-carcinogenic, anti-diabetic properties, and prevents cardiovascular diseases. Blending has improved the nutritional quality of samples as each oil has its own specificity and composition. Like, coconut oil has a high amount of medium-chain glycerides, which shows anti-obesity properties because they are easily digestible in the body; olive oil has a high amount of MUFA and therefore possess good frying properties and stability. Similarly, soybean oil is considered good for balancing lipid profile as it reduces the total and LDL cholesterol. Based on specific properties, different oils can be utilized in blends of respective oils. Although, it is still a challenge to manage SFA:MUFA:PUFA ratio as per the recommendation. Therefore, more blends of oils with different ratios can be researched to achieve the ideal composition and to improve overall quality accordingly.

\section{Funding}

This research received no external funding.

\section{Acknowledgments}

The authors acknowledge the laboratory facilities provided by the Indian Institute of Technology, Delhi, India.

\section{Conflicts of Interest}

The authors declare no conflict of interest.

\section{References}

1. Dorni, C.; Sharma, P.; Saikia, G.; Longvah, T. Fatty acid profile of edible oils and fats consumed in India. Food Chem. 2018, 238, 9-15, https://doi.org/10.1016/j.foodchem.2017.05.072. 
2. Kumar, A.; Upadhyay, N.; Padghan, P.V.; Gandhi, K.; Lal, D.; Sharma, V. Detection of vegetable oil and animal depot fat adulteration in anhydrous milk fat (ghee) using fatty acid composition. MOJ Food Process Technol 2015, 01, 01-08,https://doi.org/10.15406/mojfpt.2015.01.00013.

3. Nagaraju, A.; Belur, R.; Lokesh. Interesterified coconut oil blends with groundnut oil or olive oil exhibit greater hypocholesterolemic effects compared with their respective physical blends in rats. Nutrition Research 2007, 27, 580-586,https://doi.org/10.1016/j.nutres.2007.06.007.

4. Food Safety \& Standards Authority of India (FSSAI). Act 2006, Rule 2011.

5. Ostrikov, A. N.; Kleymenova, N. L.; Bolgova, I. N.; Kopylov, M. V. Gas chromatographic analysis of the fatty acid composition of mustard oil obtained by cold pressing (method). Emirates Journal of Food and Agriculture 2020, 32 (05), 391-396. Doi: 10.9755/ejfa.2020.v32.i5.2107.

6. Food Standards Australia New Zealand. Erucic acid in food. A toxicological review and risk assessment. Technical report series 21, 2003, ISSN: 14482-3017.

7. Kostik, V.; Memeti, S.; Bauer, B. Fatty acid composition of edible oils and fats. Journal of Hygienic Engineering and Design 2013, 4, 112-116.

8. Adeleke, B.S.; Babalola, O.O. Oilseed crop sunflower (Helianthus annuus) as a source of food: Nutritional and health benefits. Food Science \& Nutrition 2020, 8 (9), 4666-4684. https://doi.org/10.1002/fsn3.1783.

9. Matos, L.C.; Cunha, S.C.; Amaral, J.S.; Pereira, J.A.; Andrade, P.B.; Seabra, R.M.; Oliveira, B.P. Chemometric characterization of three varietal olive oils (Cvs. Cobrançosa, Madural and Verdeal Transmontana) extracted from olives with different maturation indices. Food Chem 2007, 102, 406414,https://doi.org/10.1016/j.foodchem.2005.12.031.

10. El Riachy, M.; Hamadeh, A.; Ayoub, R.; Dandachi, F.; Chalak, L. Oil content, fatty acid and phenolic profiles of some olive varieties growing in Lebanon. Frontiers in Nutrition 2019, 6, 94. Doi: 10.3389/fnut.2019.00094.

11. Jimenez-Lopez, C.; Carpena, M.; Lourenço-Lopes, C.; Gallardo-Gomez, M.; Lorenzo, J. M.; Barba, F. J.; Prieto, M.A.; Simal-Gandara, J. Bioactive compounds and quality of extra virgin olive oil. Foods 2020, 9 (8), 1014. Doi: 10.3390/foods9081014.

12. Huiling, M.U.; Carl, E.H. The digestion of dietary triacylglycerols- review. Progress in Lipid Research 2004, 43, 105-133. https://doi.org/10.1016/S0163-7827(03)00050-X.

13. Jayawardena, R.; Swarnamali, H.; Lanerolle, P.; Ranasinghe, P. Effect of coconut oil on cardio-metabolic risk: A systematic review and meta-analysis of interventional studies. Diabetes \& Metabolic Syndrome: Clinical Research \& Reviews 2020 (in press). https://doi.org/10.1016/j.dsx.2020.09.033.

14. Neelakantan, N.; Seah, J. Y. H.; van Dam, R.M. The Effect of Coconut Oil Consumption on Cardiovascular Risk Factors: A Systematic Review and Meta-Analysis of Clinical Trials. Circulation 2020, 141 (10), 803814. https://doi.org/10.1161/CIRCULATIONAHA.119.043052.

15. Medeiros de Azevedo, W.; Ribeiro de Oliveira, L.F.; Alcântara, M.A.; Tribuzy de Magalhães Cordeiro, A.M.; Florentino da Silva Chaves Damasceno, K.S.; Kelly de Araújo, N.; Fernandes de Assis, C.; Caninde de Sousa Junior, F. Physicochemical characterization, fatty acid profile, antioxidant activity and antibacterial potential of cacay oil, coconut oil and cacay butter. PloS one 2020, 15, no. 4, e0232224.

16. Harris, W.S.; Miller, M.; Tighe, A.P.; Davidson, M.H.; Schaefer, E.J. Omega- 3 fatty acids and coronary heart disease risk: Clinical and mechanistic perspectives. Atherosclerosis 2007, 197, 1224,https://doi.org/10.1016/j.atherosclerosis.2007.11.008.

17. Parikh, M.; Maddaford, T. G.; Austria, J. A.; Aliani, M.; Netticadan, T.; Pierce, G. N. Dietary flaxseed as a strategy for improving human health. Nutrients 2019, 11 (5), 1-15. Doi: 10.3390/nu11051171.

18. WHO. Interim summary of conclusions \& dietary recommendations on total fat \& fatty acids. The joint FAO/WHO expert consultation on fats \& fatty acids in human nutrition. Geneva: WHO, 2008, 1-14.

19. O'Fallon, J.V.; Busboom, J.R.; Nelson, M.L.; Gaskins, C.T. A direct method for fatty acid methyl ester synthesis: Application to wet meat tissues, oils and feedstuffs. Journal of Animal Science 2007, 85, 15111521. https://doi.org/10.2527/jas.2006-491.

20. Gruia, A.; Dwmbrava, G. D.; Moldovan, C.; Bordean, D.M. Fatty acid composition and oil characteristics of linseed oil from Romania. Journal of Agroalimentary Process and Technologies 2012, 18, 136-140.

21. Prasanth, K.; Gopala, K. Physicochemical characteristics of commercial coconut oils produced in India. Grasas y aceites 2015, 66, 62-73,https://doi.org/10.3989/gya.0228141.

22. Liu, K. Properties and Edible Applications of Soybean Oil. In: Soybeans. Springer, Boston, MA. 1997; pp. 347-378,https://doi.org/10.1007/978-1-4615-1763-4_7.

23. Abdalla, I. B. H.; Khaddor, M.; Boussab, A.; El Garrouj, D.; Souhial, B. Physical and Chemical Characteristics of Olive Oils from Cooperatives for Olive Growers in the North of Morocco. International Journal of Basic \& Applied Sciences 2014, 14, 04-11.

24. Swarnali, C.; Surashree, S.G.; Avery, S.; Mahua, G. Quality ascertain of different mustard oil samples obtained from the localmarket of West Bengal, India. Asian journal of Dairy and Food Research 2018, 37, 138-143,https://doi.org/10.18805/ajdfr.DR-1197.

25. Konuskan, D.B.; Arslan, M.; Oksuz, A. Physicochemical properties of cold pressed sunflower, peanut, rapeseed, mustard and olive oils grown in the Eastern Mediterranean region. Saudi Journal of Biological Sciences 2019, 26 (2), 340-344. https://doi.org/10.1016/j.sjbs.2018.04.005. 
26. Kumar, A.; Naik, S.N.; Gandhi, K.; Pandey, V. Functional lipid components for obesity management: a review. International Food Research Journal 2019, 26, 1111-1122.

27. Maszewska, M.; Florowska, A.; Dłuzewska, E.; Wroniak, M.; Marciniak Lukasiak, K.; Zbikowska, A. Oxidative Stability of Selected Edible Oils. Molecules 2018, 23, 17461758,https://doi.org/10.3390/molecules23071746.

28. Tagoe, S.M.A.; Dickinson, M.J.; Apetorgbor, M.M. Factors influencing quality of palm oil produced at the cottage industry level in Ghana. International Food Research Journal 2012, 19, 271-278.

29. Kumar, R.; Chandra, S.; Samsher, K.K.; Kumar, T.; Kumar, V. Analysis of the physical and chemical characteristics of edible vegetable blended oil. Internation Journal of Chemical Studies 2018, 6, 10-15.

30. Iqbal, S.; Bhanger, M. Stabilization of sunflower oil by garlic extract during accelerated storage. Food chem 2007, 100, 246-254,https://doi.org/10.1016/j.foodchem.2005.09.049.

31. Azuaga, I.C.; Abare, L.; Jen, D.B.; Ogori, B. Effect of Storage Temperatureon the Development of Rancidity by selected Vegetables Oils sold in Jalingo Main market, Taraba State-Nigeria. Chemistry research journal 2018, 3, 23-27. 